Pacific Journal of Mathematics

SPECTRAL SYNTHESIS IN SOME SPACES OF BOUNDED 


\title{
SPECTRAL SYNTHESIS IN SOME SPACES OF BOUNDED CONTINUOUS FUNCTIONS
}

\author{
AHARON ATZMON
}

\begin{abstract}
Spectral synthesis in the topology of bounded uniform convergence on compact sets is proved for some spaces of bounded continuous functions on the real line $R$. These spaces include among others the space of continuous functions of bounded variation on $R$ and the space of bounded functions on the real line which are linear combinations of convex functions which satisfy Lipshitz condition of order one uniformly on $R$.
\end{abstract}

o. Introduction. In the extensive and varied literature which appeared on the subject of spectral synthesis in $L^{\infty}(R)$, mainly after Malliavin's disproof of spectral synthesis in general [7] the main emphasis was stressed on the study of sets which obey and sets which disobey spectral synthesis. On the other hand, relatively little attention was paid to the investigation of classes of $L^{\infty}(R)$ functions which admit spectral synthesis. A discussion of the main results which exist in this direction can be found in [1].

The purpose of this paper is to prove for some classes of bounded continuous functions on the real line, that spectral synthesis holds in the topology of bounded uniform convergence on compact sets.

In order to state our main result we need first some notations. For every positive integer $n$ let $\Delta^{n}$ denote the $n$th difference operator defined inductively on two sided sequences $\left(a_{k}\right)_{k=-\infty}^{\infty} b y: \Delta^{1} a_{k}=a_{k}-a_{k-1}$, $k=0, \pm 1, \pm 2, \cdots$, and for $n \geqq 1, \Delta^{n} a_{k}=\Delta^{1}\left(\Delta^{n-1} a_{k}\right), k=0, \pm 1, \pm 2, \cdots$. The convention $\Delta^{0} a_{k}=a_{k}, k=0, \pm 1, \pm 2, \cdots$, will also be adopted.

Our main result is the following:

THEOREM 1. Let $\phi$ be a bounded cantinuous function on $R$ such that for some nonnegative integer $n$ and some real number $1 \leqq p \leqq 2$, the condition

$$
\sup _{N} \varlimsup_{h \rightarrow 0+} \sum_{|k| \leq N / h} \frac{\left|\Delta^{n} \phi(k h)\right|^{p}}{h^{n p-1}}<\infty
$$

is satisfied. Then $\phi$ admits spectral synthesis in the topology of bounded uniform convergence on compact sets.

Since the continuous functions on $R$ which satisfy condition (1.1) for $n=p=1$ are exactly the continuous functions of bounded variation on $R$, it follows from this theorem that in the conclusion of Theorem 1 in [1] the $w^{*}$-topology can be replaced by the stronger 
topology of bounded uniform convergence on compact sets if continuity is added to its hypotheses. Another concrete application of this theorem is in the case $n=2$ and $p=1$. It will follow from the general characterization given in $\S 2$ of the bounded continuous functions on $R$, which satisfy condition (1.1) for some $n$ and $p$, that a bounded continuous function $\phi$ on $R$ satisfies condition (1.1) for $n=2$ and $p=1$ if and only if $\phi$ is a linear combination of four convex functions which satisfy Lipshitz condition of order one uniformly on $R$. Hence by Theorem 1 such functions admit bounded spectral synthesis in the topology of bounded uniform convergence on compact sets.

1. Basic definitions. We recall now some basic definitions concerning spectral synthesis of bounded functions. For further details on spectral synthesis we refer to $[3$, p. 547], [5, p. 170] and [8, p. 183].

Let $\phi$ be a bounded continuous complex function on the real line $R$. For every real number $y$, the $y$-translate of $\phi$ is the function $\phi_{y}$ defined by $\phi_{y}(x)=\phi(x-y), x \in R$. The $w^{*}$-spectrum of $\phi$ is the set $\mathrm{sp} \phi$ of real numbers $\lambda$ such that the function $e^{i \lambda x}$ is in the $w^{*}$ closed subspace of $L^{\infty}(R)$ which is spanned by the translates of $\phi$.

Let $M(R)$ denote the space of bounded complex Borel measures on $R$. For every measure $\mu$ in $M(R)$ let $\hat{\mu}$ denote its Fourier Stieltjes transform defined by $\hat{\mu}(x)=\int_{R} e^{i x t} d \mu(t), x \in R$.

One says that the bounded continuous function $\phi$ on $R$, admits spectral synthesis in the $w^{*}$-topology, if there exists a net $\left(\mu_{\gamma}\right)_{\gamma \in \Gamma}$ in $M(R)$ such that: $\operatorname{sp} \hat{\mu}_{r} \subset \operatorname{sp} \phi, \gamma \in \Gamma$ and $\lim _{r} \hat{\mu}=\phi$ in the $w^{*}$-topology of $L^{\infty}(R)$.

One says that $\phi$ admits bounded spectral synthesis in the topology of uniform convergence on compacta, if there exists a sequence $\left(\mu_{n}\right)_{n=1}$ in $M(R)$ such that: $\operatorname{sp} \hat{\mu}_{n} \underline{c} \operatorname{sp} \phi, n=1,2, \cdots, \sup _{n}\left\|\hat{\mu}_{n}\right\|_{\infty}<\infty$, and $\lim \hat{\mu}_{n}(x)=\phi(x)$ uniformly on compact subsets of $R$.

Spectral synthesis in the topology of bounded uniform convergence on compact sets clearly implies spectral synthesis in the $w^{*}$ topology. That the converse is not true for all bounded continuous functions, follows from [10, Thm. 3].

2. The spaces $B_{n, p *}$ For a bounded continuous function $\dot{\phi}$ on $R$, a nonnegative integer $n$ and a real number $p \geqq 1$, we denote by

$$
\|\dot{\phi}\|_{n, p}=\left(\sup _{N} \varlimsup_{h \rightarrow 0+} \sum_{|k| \leq N / h} \frac{\left|\Delta^{n} \phi(k h)\right|^{p}}{h^{n p-1}}\right)^{1 / p}
$$

and by $B_{n, p}$ the space of bounded continuous functions $\phi$ on $R$ such 
that $\|\phi\|_{n, p}$ is finite. It can be shown that with norm $\|\phi\|_{\infty}+\|\phi\|_{n, p}$ the spaces $B_{n, p}$ are Banach spaces. This fact will not be used in the sequel.

Theorem 1 can now be stated in the following equivalent form:

THeOREM $1^{\prime}$. If $n$ is a nonnegative integer and $1 \leqq p \leqq 2$ then every function $\phi$ in $B_{n, p}$ admits spectral synthesis in the topology of bounded uniform convergence on compact sets.

It is obvious that $B_{0, p}$ consists of all bounded continuous functions which are in $L^{p}(R)$ and $B_{1,1}$ is the space of continuous functions which are of bounded variation on $R$. Also $B_{2,1}$ consists of all bounded continuous functions on $R$ which are linear combinations of four convex functions which satisfy Lipshitz condition of order 1 uniformly on $R$. This follows easily from the characterization of the spaces $B_{n, p}$ given in Lemma 1 below. This lemma is needed for the proof of Theorem 1, and is also of some interest in itself.

LeMma 1. Let $\phi$ be a bounded continuous function on $(-\infty, \infty)$. For a nonnegative integer $n$ let $\phi^{(n)}$ denote the $n$th derivative of $\phi$ in the sense of distributions. Then:

(a) $\phi$ is in $B_{n, 1}$, for $n \geqq 1$, if and only if $\dot{\phi}^{(n)}$ is a bounded Borel measure on $R$. (As already remarked, $\phi$ is in $B_{0,1}$ if and only if $\phi$ is in $L^{1}(R)$.)

(b) If $1<p<\infty$ then $\phi$ is in $B_{n, p}(n=0,1,2, \cdots)$ if and only if $\dot{\phi}^{(n)}$ is in $L^{p}(R)$.

Proof (a). Let $D$ denote the space of infinitely differentiable functions on $R$ with compact support. Consider $\phi$ in $B_{n, 1}$ and $\psi$ in $D$. Let $N$ be a positive integer such that the support of $\psi$ is contained in the interval $[-N, N]$. Since $\phi$ is continuous and $\psi$ is in $D$, an elementary argument implies that

$$
\lim _{h \rightarrow 0+} \sum_{|k| \leqq N / h} \frac{\Delta^{n} \psi(k h+n h)}{h^{n-1}} \dot{\phi}(k h)=\int_{-\infty}^{\infty} \psi^{(n)}(x) \phi(x) d x
$$

where $\psi^{(n)}$ denotes of course the $n$th derivative of $\psi$ in the ordinary sense.

Summation by parts yields:

$$
\sum_{|k| \leqq N / h} \frac{\Delta^{n} \psi(k h+n h)}{h^{n-1}} \phi(k h)=(-1)^{n} \sum_{|k| \leqq N / h} \psi(k h) \frac{\Delta^{n} \phi(k h)}{h^{n-1}}
$$

and therefore by (2.1) 


$$
\left|\int_{-\infty}^{\infty} \phi(x) \psi^{(n)}(x) d x\right| \leqq\|\phi\|_{n, 1}\|\psi\|_{\infty}
$$

That is, the linear functional defined on $D$ by $\psi \rightarrow \int_{-\infty}^{\infty} \phi(x) \psi^{(n)}(x) d x$, $\psi \in D$, is bounded in the sup-norm. Consequently (cf. 9, p. 25) there exists a bounded Borel measure $\mu$ on $R$ such that

$$
\int_{-\infty}^{\infty} \phi(x) \psi^{(n)}(x) d x=(-1)^{n} \int_{R} \psi(x) d \mu(x), \text { for every } \psi \text { in } D
$$

and therefore $\phi^{(n)}=\mu$ in the sense of distributions.

To prove the converse, assume that $\phi^{(n)}=\mu$ for some bounded Borel measure $\mu$ on $R$. Consider the Fejér Kernel

$$
K_{j}(x)=\frac{1}{2 \pi j}\left(\frac{\sin j \frac{x}{2}}{x / 2}\right)^{2}, j=1,2, \cdots,(\text { see } 5, \text { p. 124), }
$$

and the sequence of bounded continuous functions $\phi_{j}=K_{j} * \phi,(j=1,2, \cdots)$ (that is $\left.\phi_{j}(x)=\int_{-\infty}^{\infty} K_{j}(x-y) \phi(y) d y,-\infty<x<\infty\right)$. Since $K_{j}$ is a $C^{\infty}$ function all whose derivatives are in $L^{1}(R)$ it follows that $\phi_{j}$ is also a $C^{\infty}$ function and $\phi_{j}^{(n)}=\left(K_{j} * \phi\right)^{(n)}=K_{j} * \phi^{(n)}=K_{j} * \mu$, where $\phi_{j}^{(n)}$ denotes the $n$th derivative of $\phi_{j}$ in the usual sense and $\phi^{(n)}$ the $n$th derivative of $\phi$ in the sense of distributions.

Therefore, $\left\|\dot{\phi}_{j}^{(n)}\right\|_{1} \leqq\left\|K_{j}\right\|_{1}\|\mu\|=\|\mu\| j=1,2, \cdots$, where $\|\mu\|$ denotes the total variation of $\mu$. Hence for every positive integer $N$,

$$
\lim _{h \rightarrow 0+} \sum_{|k| \leqq N / h} \frac{\left|\Delta^{n} \dot{\phi}_{j}(k h)\right|}{h^{n-1}}=\int_{-N}^{N}\left|\phi_{j}^{(n)}(x)\right| d x \leqq\|\mu\| \quad j=1,2, \cdots
$$

Since $\phi$ is continuous, it follows from the well known properties of the Fejér Kernel that $\lim _{j \rightarrow \infty} \phi_{j}(x)=\phi(x)$ uniformly on compact sets on $R$ (cf. 5, p. $130 \mathrm{Ex} .9$ ), and therefore (2.4) implies that

$$
\varlimsup_{h \rightarrow 0+} \sum_{|k| \leq N / h} \frac{\left|\Delta^{n} \phi(k h)\right|}{h^{n-1}} \leqq\|\mu\|
$$

for all positive integers $N$. This completes the proof of (a).

Proof (b). The proof of (b) is similar to that of (a). Let $1<$ $p<\infty$ and $q=p /(p-1)$. Consider $\phi$ in $B_{n, p}$ and $\psi$ in $D$ with support in $[-N, N]$. Using (2.2) and Hölder's inequality we infer that

$$
\left|\sum_{|k| \leqq N / h} \frac{\Delta^{n} \psi(k h+n h)}{h^{n-1}} \phi(k h)\right| \leqq\left(\left.\sum_{|k| \leqq N / h} \frac{\Delta^{n} \dot{\phi}(k h)}{h^{n p-1}}\right|^{p}\right)^{1 / p}\left(\sum_{|k| \leqq N / h}|\psi(k h)|{ }^{q} h\right)^{1 / q} .
$$

Hence passing to $\varlimsup$ as $h \rightarrow 0+$ we obtain as in (2.1) that 


$$
\left|\int_{-\infty}^{\infty} \phi(x) \psi^{(n)}(x) d x\right| \leqq\|\phi\|_{n, p}\|\psi\|_{q} \text {. }
$$

Since (2.5) holds for every $\psi$ in $D$, a standard duality argument (as in the proof of (a)) implies that there exists a function $g \in L^{p}(R)$ such that

$$
\int_{-\infty}^{\infty} \phi(x) \psi^{(n)}(x) d x=(-1)^{n} \int_{-\infty}^{\infty} \phi(x) g(x) d x
$$

for every $\psi$ in $D$. Consequently $\psi^{(n)}=g$ in the sense of distributions.

To prove the converse of (b) the same argument as in the corresponding part of (a) is used, and we omit the details. This completes the proof of the lemma.

The next lemma gives more insight into the structure of the spaces $B_{n, p}$ for $n \geqq 2$, and in particular implies the characterization of the spaces $B_{2,1}$ already mentioned in $\S 0$ and at the beginning of this section.

Lemma 2. Let $n \geqq 2$ be an integer. Then

(I) $\phi$ is in $B_{n, 1}$ if and only if $\phi$ has $n-2$ continuous derivatives on $R, \phi^{(n-2)}$ is absolutely continuous and $\phi^{(n-1)}$ is a function of bounded variation on $R$.

(II) If $1<p<\infty$ then $\phi$ is in $B_{n, p}$ if and only if $\phi$ has $n-1$ continuous derivatives on $R, \phi^{(n-1)}$ is absolutely continuous and $\phi^{(n)}$ is in $L^{p}(R)$.

This lemma follows easily from Lemma 1 (see also 9, p. 53 and p. 54), and since it is not used in the sequel we omit the proof.

We are now in position to give the

Proof of Theorem 1. Let $\phi$ be in $B_{n, p}$ for some nonnegative integer $n$ and some $1 \leqq p \leqq 2$. We show first that the proof of the theorem can be reduced to the case in which $\mathrm{sp} \phi$ is compact. This can be seen as follows:

Lemma 1 implies that for every $K$ in $L(R)$ the function $K * \phi$ is in $B_{n, p}$. Hence, denoting as before by $K_{j}, j=1,2, \cdots$, the Fejér Kernel, it follows that the sequence $K_{j} * \phi, j=1,2, \cdots$, is in $B_{n, p}$. Since $\left\|K_{j} * \dot{\phi}\right\|_{\infty} \leqq\left\|K_{j}\right\|_{1}\|\phi\|_{\infty}=\|\phi\|_{\infty}, j=1,2, \cdots$, and $\lim _{j \rightarrow \infty} K_{j} * \dot{\phi}(x)=$ $\phi(x)$ uniformly on compact sets, we infer that if for every $j=1,2, \cdots$, the function $K_{j} * \phi$ admits spectral synthesis in the topology of bounded uniform convergence on compact sets, the same will be true for $\phi$. But the fact that the support of the Fourier transform of $K_{j}$ is compact implies [5, p. 170] that $\mathrm{sp}\left(K_{j} * \phi\right)$ is compact. Consequently, we may assume, as we shall do in the sequel, that $\mathrm{sp} \phi$ 
is compact.

For every positive integer $j$ let $V_{j}$ denote the function in $L^{1}(R)$ whose Fourier transform $\hat{V}_{j}$ is 1 on $[-j, j], 0$ outside $[-2 j, 2 j]$, and linear elsewhere. It is known [5, p. 136] that $\left\|V_{j}\right\|_{1} \leqq 3, j=$ $1,2, \cdots$. Let $W_{j}$ be the function defined by

$$
W_{j}(x)=V_{j}(x)-\frac{1}{j^{2}} V_{j}\left(\frac{x}{j^{2}}\right), \quad-\infty<x<\infty, \quad(j=1,2, \cdots) .
$$

It follows easily from the properties of $V_{j}$ that $\left\|W_{j}\right\|_{1} \leqq 6$ and that $\hat{W}_{j}(x)=0$ for $|x| \leqq 1 / 2 j$ and $|x| \geqq 2 j$, and $\hat{W}_{j}(x)=1$ for $1 / 2 j \leqq|x| \leqq j$. Let $\psi_{j} \equiv W_{j} * \phi, j=1,2, \cdots$; then $\left\|\psi_{j}\right\|_{\infty} \leqq\left\|W_{j}\right\|_{1}\|\dot{\phi}\|_{\infty} \leqq 6\|\phi\|_{\infty}$. We claim now that the sequence $\left(\psi_{j}\right)_{j=1}^{\infty}$ is equicontinuous. In fact, let $K$ be in $L^{1}(R)$ such that $\hat{K}=1$ on a neighborhood of $\operatorname{sp} \phi$ (which we assume to be compact). Since $\mathrm{sp}\left(K_{j} * \phi\right) \subseteq \mathrm{sp} \phi[5, \mathrm{p} .152$ and p. 170] we obtain [5, p. 152] that $K * \psi_{j}=\psi_{j}, j=1,2, \ldots$. For every real number $\tau$, let $K_{\tau}$ denote the function defined by $K_{\tau}(x)=K(x-\tau)$, $-\infty<x<\infty$. Then for every real $x$ and $h$ we have:

$$
\begin{aligned}
& \left|\psi_{j}(x-h)-\psi_{j}(x)\right|=\left|K * \psi_{j}(x-h)-K * \psi_{j}(x)\right| \\
& \quad \leqq\left\|K_{h}-K\right\|_{1}\left\|\psi_{j}\right\|_{\infty} \leqq 6\|\phi\|_{\infty}\left\|K_{h}-K\right\|_{1}, \quad j=1,2, \cdots,
\end{aligned}
$$

and since $\lim _{h \rightarrow 0}\left\|K_{h}-K\right\|_{1}=0$, the sequence $\left(\psi_{j}\right)_{j=1}^{\infty}$ is equicontinuous. Since also $\sup _{j}\left\|\psi_{j}\right\|_{\infty}<\infty$, the theorem of Arzela-Ascoli implies that there exists a subsequence $\left(\psi_{j_{k}}\right)_{k=1}^{\infty}$ which converges uniformly on compact sets to some bounded continuous function $\psi$. Since $\hat{W}_{j}(x)=1$ for $1 / j \leqq|x| \leqq j$ and $\hat{\psi}_{j}=\hat{W}_{j} \cdot \hat{\phi}$ (the Fourier transforms are in the sense of distributions) it follows $[5, \mathrm{p} .170]$ that $\operatorname{sp}(\phi-\psi) \cong\{0\}$, (that is, $s p(\phi-\psi)$ is empty or consists of the single point 0$)$. Therefore by a well known result on the structure of bounded functions with one point spectrum [5, p. 136] it follows that $\phi=\psi+c$ where $c$ is a constant, (which is zero if $\operatorname{sp}(\phi-\psi)$ empty). Therefore denoting by $\phi_{k}=\psi_{j_{k}}+c, k=1,2, \cdots$, we have that: $\sup _{k}\left\|\phi_{k}\right\|_{\infty}<\infty$ and $\lim _{k \rightarrow \infty} \phi_{k}(x)=\phi(x)$ uniformly on compact sets. Moreover, from the fact that $\operatorname{sp} \psi_{j} \subseteq \operatorname{sp} \phi, j=1,2, \cdots$, it follows that $\mathrm{sp} \psi \subseteq \mathrm{sp} \phi$, and this implies that $\operatorname{sp}_{k} \subseteq \operatorname{sp} \phi, k=1,2, \cdots$. This is clear if $c=0$, and if $c \neq 0$ then $0 \in \mathrm{sp}(\phi-\psi) \leqq \mathrm{sp} \phi$, and therefore $\operatorname{sp} \phi_{k}=\operatorname{sp}\left(\psi_{j_{k}}+c\right) \subseteq$ $\operatorname{sp} \phi, k=1,2, \cdots$. Consequently, the proof of the theorem will be accomplished by showing that for every $k=1,2, \cdots$, the function $\phi_{k}$ is a Fourier Stieltjes transform of a bounded Borel measure. This fact will be proved by showing that for every $j=1,2, \cdots, \psi_{j}$ is a Fourier transform of an $L^{1}(R)$ function.

By Lemma $1, \phi^{(n)}=\nu$ where $\nu$ is a bounded Borel measure if $p=1$ and an $L^{p}(R)$ function if $1<p \leqq 2$. Therefore, taking Fourier transforms in the sense of distributions we get $[9$, p. 253] that 
$\hat{\phi}(x)=\hat{\nu}(x) /(i x)^{n}$, where $\hat{\nu}$ is a Fourier Stieltjes transform if $p=1$, and if $p>1, \hat{\nu}$ is an $L^{q}(R)$ function where $q=p /(p-1)$, by the Hausdorff-Young theorem. Hence remembering that $\hat{W}_{j}(x)=0$ for $|x| \leqq 1 / 2 j$ and $|x| \geqq 2 j$, and that $\hat{\psi}_{j}=\hat{W}_{j} \cdot \hat{\phi}$, it follows that $\hat{\psi}_{j}$ is in $L^{1}(R)$ in both cases $(p=1$ and $1<p \leqq 2)$. Therefore by the inversion theorem $\psi_{j}$ is a Fourier transform of an $L^{1}(R)$ function and the theorem is proved.

4. Extensions and sharpness of results. The proof of Theorem 1 can be clearly extended to include functions which are linear combinations of functions in the spaces $B_{n, p}$ for $n=0,1,2, \cdots$, and $1 \leqq p \leqq 2$. More precisely the following generalization of Theorem 1 holds:

THeOREM 2. Let $M$ be the linear span in $L^{\infty}(R)$ of the union of the spaces $B_{n, p}, n=0,1,2, \cdots, 1 \leqq p \leqq 2$. Then every function $\phi$ in $M$, admits spectral synthesis in the topology of bounded uniform convergence on compact sets.

It is perhaps worthwhile to remark here that it is not true in general that the sum of two functions each of which admits spectral synthesis in the topology of bounded uniform convergence on compact sets, admits spectral synthesis even in the $w^{*}$-topology. Such an example can be constructed by using the existence of a bounded continuous function $\phi$ on $R$ which does not admit spectral synthesis in the $w^{*}$-topology (Malliavin's theorem) and a result of C. Herz [4, p. $229 \mathrm{Thm}$. 6.5] which implies (cf. 6, p. $124 \mathrm{Thm}$. V) that for every compact set $E$ in $R$ there exists a countable set $F$ such that every bounded continuous function $\phi$ on $R$ such that $\operatorname{sp} \phi=E \cup F$ admits spectral synthesis in the topology of bounded uniform convergence on compact sets. We omit the details.

Finally we show that the condition $1 \leqq p \leqq 2$ in Theorem 1 , cannot be replaced by $1 \leqq p<\infty$. That is the following holds

Proposition. For every nonnegative integer $n$ and every $p>2$, there exists a function $\phi$ in $B_{n, p}$, which does not admit spectral synthesis even in the $w^{*}$-topology.

Proof. It is known that for every $p>2$ there exists a bounded continuous function $\phi$ in $L^{p}(R)$ such that $\operatorname{sp} \phi$ is compact, which does not admit spectral synthesis in the $w^{*}$-topology. This result does not appear explicitly in the literature but follows easily from [6, p.121] where the corresponding result for the group of integers is proved. The passage to $R$, and in fact to general locally compact 
abelian groups which are not compact, is outlined in [2, p. 247] in a different context. Hence for $p>2$, there exists a function $\phi$ in $B_{0, p}$ with compact spectrum, which does not admit spectral synthesis in the $w^{*}$-topology. We claim that this function is in $B_{n, p}$ for every positive integer $n$. In fact, let $K$ be a $C^{\infty}$ function in $L^{1}(R)$ such that $K^{(n)}$ is also in $L^{1}(R)$ and such that $\hat{K}=1$ on a neighborhood of $\operatorname{sp} \phi$. Then $\phi=K * \phi$ and therefore $\phi^{(n)}=K^{(n)} * \phi$ is also in $L^{p}(R)$, as a convolution of an $L^{1}(R)$ function with an $L^{p}(R)$ function. Hence $\phi$ is in $B_{n, p}$ and the proposition is proved.

\section{REFERENCES}

1. A. Atzmon, Spectral synthesis of functions of bounded variation, Proc. Amer. Math. Soc., 47 (1975), 417-422.

2. - Translation invariant subspaces of $L^{p}(G)$, Studia Math., XLIII (1973), 245-250.

3. E. Hewitt and K. Ross, Abstract harmonic analysis, vol. II, Springer 1970.

4. C. S. Herz, The spectral theory of bounded functions, Trans. Amer. Math. Soc., 94 (1960), 181-232.

5. Y. Katznelson, An Introduction to Harmonic Analysis, Wiley 1968.

6. J. P. Kahane et R. Salem, Ensembles parfaits et séries trigonométriques, HermanParis 1963.

7. P. Malliavin, Sur l'impossibilité de la synthèse spectrale sur la droite, C. R. Acad.

Sc. Paris, 248 (1959), 1756-1759.

8. W. Rudin, Fourier Analysis on Groups, Interscience 1962.

9. L. Schwartz, Théorie des distributions, Herman-Paris 1966.

10. N. Th. Varopoulos, Groups of continuous functions in harmonic analysis, Acta Math., 125 (1970), 109-154.

Received June 9, 1977.

Technion, Haifa, Israel 


\section{PACIFIC JOURNAL OF MATHEMATICS}

\section{EDITORS}

RICHARD ARENS (Managing Editor)

University of California

Los Angeles, CA 90024

Charles W. Curtis

University of Oregon

Eugene, OR 97403

C. C. Moore

University of California

Berkeley, CA 94720

\section{J. DugundJI}

Department of Mathematics

University of Southern California

Los Angeles, CA 90007

R. FInN and J. Milgram

Stanford University

Stanford, CA 94305

\section{ASSOCIATE EDITORS}
E. F. BECKENBACH
B. H. NeumanN
F. WOLF
K. YoSHIDA

\section{SUPPORTING INSTITUTIONS}

\author{
UNIVERSITY OF BRITISH COLUMBIA \\ CALIFORNIA INSTITUTE OF TECHNOLOGY \\ UNIVERSITY OF CALIFORNIA \\ MONTANA STATE UNIVERSITY \\ UNIVERSITY OF NEVADA, RENO \\ NEW MEXICO STATE UNIVERSITY \\ OREGON STATE UNIVERSITY \\ UNIVERSITY OF OREGON
}

\author{
UNIVERSITY OF SOUTHERN CALIFORNIA \\ STANFORD UNIVERSITY \\ UNIVERSITY OF HAWAII \\ UNIVERSITY OF TOKYO \\ UNIVERSITY OF UTAH \\ WASHINGTON STATE UNIVERSITY \\ UNIVERSITY OF WASHINGTON
}

The Supporting Institutions listed above contribute to the cost of publication of this Journal, but they are not owners or publishers and have no responsibility for its content or policies.

Mathematical papers intended for publication in the Pacific Journal of Mathematics should be in typed form or offset-reproduced, (not dittoed), double spaced with large margins. Please do not use built up fractions in the text of the manuscript. However, you may use them in the displayed equations. Underline Greek letters in red, German in green, and script in blue. The first paragraph or two must be capable of being used separately as a synopsis of the entire paper. Items of the bibliography should not be cited there unless absolutely necessary, in which case they must be identified by author and journal, rather than by item number. Manuscripts, in triplicate, may be sent to any one of the editors. Please classify according to the scheme of Math. Reviews, Index to Vol. 39. All other communications should be addressed to the managing editor, or Elaine Barth, University of California, Los Angeles, California, 90024.

50 reprints to each author are provided free for each article, only if page charges have been substantially paid. Additional copies may be obtained at cost in multiples of 50 .

The Pacific Journal of Mathematics is issued monthly as of January 1966. Regular subscription rate: $\$ 72.00$ a year (6 Vols., 12 issues). Special rate: $\$ 36.00$ a year to individual members of supporting institutions.

Subscriptions, orders for numbers issued in the last three calendar years, and changes of address should be sent to Pacific Journal of Mathematics, 103 Highland Boulevard, Berkeley, California, 94708. Older back numbers obtainable from Kraus Periodicals Co., Route 100, Millwood, NY 10546.

PUBLISHED BY PACIFIC JOURNAL OF MATHEMATICS, A NON-PROFIT CORPORATION

Printed at Kokusai Bunken Insatsusha (International Academic Printing Co., Ltd.). 8-8, 3-chome, Takadanobaba, Shinjuku-ku, Tokyo 160, Japan.

Copyright (C) 1978 by Pacific Journal of Mathematics

Manufactured and first issued in Japan 


\section{Pacific Journal of Mathematics

Vol. 74, No. $2 \quad$ June, 1978

Aharon Atzmon, Spectral synthesis in some spaces of bounded continuous

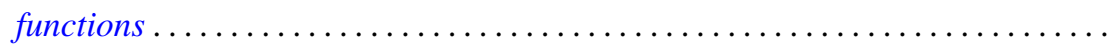

Karl Egil Aubert and Isidor Fleischer, Tensor products of ideal systems and their modules.............................................

Richard F. Basener, Several dimensional properties of the spectrum of a uniform

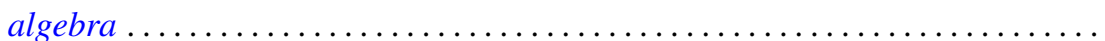

R. H. Bing and Michael Peter Starbird, Super triangulations ............. 307

Andrew Carson, Coherent polynomial rings over regular rings of finite index .....

Robert M. DeVos and Frederick W. Hartmann, Sequences of bounded summability domains .................................................

George Grätzer and R. Padmanabhan, Symmetric difference in abelian groups ....

Robert L. Griess, Jr., A remark about groups of characteristic 2-type and

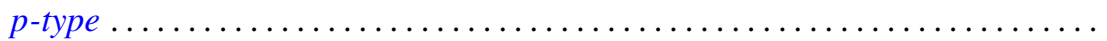

Emil Grosswald and F. J. Schnitzer, A class of modified $\zeta$ and L-functions........

Jutta Hausen and Johnny Albert Johnson, Ideals and radicals of some

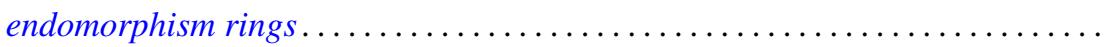

Jean Ann Larson, A solution for scattered order types of a problem of Hagendorf ............................................

Peter A. McCoy, Extremal properties of real biaxially symmetric potentials in $E^{2(\alpha+\beta+2)}$

Héctor Alfredo Merklen, Hereditary crossed product orders .

Hal G. Moore and Adil Mohamed Yaqub, Equational definability of addition in certain rings...

Robert Laurens Moore, Reductivity in $C^{*}$-algebras and essentially reductive operators. . .

Joseph Alvin Neisendorfer, Lie algebras, coalgebras and rational homotopy theory for nilpotent spaces...

William Raymond Nico, Bounded monoids

Richard Paul Osborne, Simplifying spines of 3-manifolds ...

Richard Paul Osborne, The simplest closed 3-manifolds. With an appendix by Osborne and J. Yelle.

Clayton Collier Sherman, The $K$-theory of an equicharacteristic discrete valuation ring injects into the $K$-theory of its field of quotients.... .

Mitchell Herbert Taibleson, The failure of even conjugate characterizations of $H^{1}$

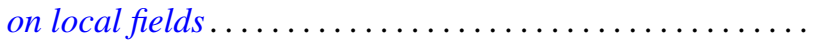

Keti Tenenblat, On characteristic hypersurfaces of submanifolds in Euclidean space ...................................

Jeffrey L. Tollefson, Involutions of Seifert fiber spaces..........

Joel Larry Weiner, An inequality involving the length, curvature, and torsions of a curve in Euclidean $n$-space .......................

Neyamat Zaheer, On generalized polars of the product of abstract homogeneous polynomials.... 